\title{
Ferromagnets-Based Multifunctional Nanoplatform for Targeted Cancer Therapy
}

\author{
Valentyn Novosad ${ }^{1}$ and Elena A. Rozhkova ${ }^{2}$ \\ ${ }^{1}$ Materials Science Division, \\ ${ }^{2}$ Center for Nanoscale Materials, \\ Argonne National Laboratory, \\ USA
}

\section{Introduction}

Nanotechnology emerges from the physical, chemical, biological, and engineering sciences, where novel techniques are being developed to probe and manipulate single atoms and molecules. While significant progress has been made in materials science, it is apparent that nanoscience and nanotechnology-based approaches are poised to revolutionize many research and technology areas. In particular, novel magnetic nanoplatforms with dimensions of few nanometers to few microns have great potential for application in biomedical sciences. Main approaches of particular clinical relevance include magnetic separation technologies, magnetic resonance imaging (MRI) contrast enhancement, targeted drug delivery, and magnetically induced hyperthermia.

In this chapter we describe the fabrication process, magnetic behavior, biofunctionalization, as well as targeted destruction of cancer cells in vitro using multifunctional ferromagnetic microdisks. The microdisks posses unique properties such as high magnetization of saturation, zero remanence due to spin vortex formation, intrinsic spin resonance at low frequencies, and capability of delivering more than one type of bio-substance at once. Furthermore, because of the anisotropic shape, lithographically-defined magnetic particles oscillate under application of alternative magnetic field of small amplitude. This phenomenon is further advanced as a new anticancer therapy approach by the application of an unprecedentedly slow and weak magnetic fields. We show that successful interfacing of lithographically defined ferromagnetic materials with a spin vortex ground state with cancer cells can be achieved in vitro. When an alternative magnetic field is applied the magnetic vortices shift, leading to oscillatory motion of the disks causing the magneto-mechanic stimulus is to be transmitted directly to the cell membrane and then into sub-cellular compartments. Such magnetic spin vortexinduced magneto-mechanical stimulus results into (a) cancer cell membrane pertrubation, as well as into (b) cellular signal transduction and amplification, causing initiation of apoptosis, a type of a programmed cell death. Manifestation of apoptosis is of clinical significance because the malignant cells are known to be almost "immortal" (due to suppressed apoptosis), and, consequently, highly resistant to conventional therapies. 


\section{Magnetic nanoparticles for biomedical applications}

\subsection{Traditional approach}

Small magnetic objects (nanoparticles) have been actively explored for application in biomedicine (Pankhurst et al., 2009). This technology is based on the ability of biological substances (cells or bio-molecules) to form molecular superstructures when they are mixed with magnetic particles, and especially valuable in the field of nanomedicine (Ferrari, 2005). In principle, selective adhesion is possible if the surface of the particle is covered by a special layer, such as gold (core-shell structure) functionalized with ligands capable of biological recognition. Here, the magnetic nature of the particle is used as a transduction mechanism for the target-directed delivery, detection, manipulation, and functional control of attached biomolecules and cells. The future of this new area of research greatly depends on progress with (1) incorporating multiple functionalities properties within a single nanoplatform, including targeting, enhanced anticancer therapy, drug delivery, imaging and detection capabilities, and (2) preparing the particles with superior magnetic properties (Nel, et al., 2009).

Major efforts up to date have been directed to synthesize and explore magnetic particles prepared by solid, gas, and solution synthesis methods. Interested readers may refer to a number of excellent reviews for the details (Krishnan, 2010; Gao, et al., 2009; Goya et al., 2008; Gupta, \& Gupta, 2005). The solid route involves the mechanical milling of powder materials to produce nanoparticles. Contamination introduced during the process as well as difficulties with controlling the particles size distribution are major drawbacks of this otherwise straight forward and low cost approach. In gas and chemical methods, the nanoparticles are synthesized through phase- conversion or phase build-up. In the first case scenario thermal decomposition or chemical reduction is employed. The phase-build-up process occurs in the gas phase (chemical or physical vapor deposition) or in the liquid phase (chemical precipitation). These techniques produce uniform nanoparticles of different shapes by using appropriate precursors and capping ligands during the reaction. Surfactant and various capping layers also help to prevent aggregation of the particles. The magnetic nanoparticles $(\sim 10 \mathrm{~s} \mathrm{~nm})$ typically are superparamagnetic. In final application they are often dispersed in, layered onto, or coated with a matrix to form beads $\sim 1 \mu \mathrm{m}$ in diameter. Known challenges related to the traditional approach include difficulty in controlling the growth process, broad size and shape distributions, and variable structural and magnetic properties. Because of their superparamagnetic nature and low value of the magnetization of saturation, high magnetic fields are required to detect or manipulate these particles. Conversely, if highly magnetic particles are synthesized (that is, larger particles with single domain spin arrangement), agglomeration may occur owing to magnetic fringe fields. On the other hand when a magnet is confined to two dimensions, the spin arrangement often takes on an interesting pattern known as a spin vortex state (Cowburn, et al., 1999; Shinjo, et al., 2000). This system can be scaled down to sub-100nm lateral dimensions with preserving closed flux spin structure and magnetic properties. Such novel magnetic particles can be fabricated using thin film growth techniques, combined with mask-transfer lithography.

\subsection{Lithographically defined particles}

Microfabrication methods were recently used to synthesize magnetic particles suitable for biomedical applications. By combining physical vapor deposition techniques (such as magnetron sputtering or electron beam evaporation) with optical, nano-imprint or laser interferometer lithography it is possible to produce monodisperse particles of virtually any 
shape, multicomponent and multilayered, with tunable magnetic properties and size down to $\sim 100 \mathrm{~nm}$. Such magnetic materials are used in giant magnetoresistance spin valve or magnetic tunnel junction sensors capable to detect minute concentrations (down the picomolar) of DNA and proteins in vitro (Wang, \& Li, 2008; Fu et al., 2009), as advanced MRI enhancement agents (Zabow, et al., 2008; Zabow, et al., 2009) and in magnetofection experiments (Mair, et al., 2009). In brief, the main advantages of the lithographically defined particles can be outlined as follows:

- Multifunctional \& scalable, biocompatible nanoplatform;

- Zero remanence (disks will not agglomerate during delivery process ;

- High saturation magnetization can be achieved using 3d metal alloys;

- Strong magneto-mechanical coupling - new way to destroy targeted cells via initiation of the intracellular biochemical signaling leading to apoptosis;

- Vortex resonant properties: possibilities for high sensitivity ac detection;

- Excellent MRI enhancement capacity;

- Targeted drug release is possible via magneto-mechanical actuation;

- $\quad$ The disks show inductive heating when subjected to $100 \mathrm{~s} \mathrm{kHz}$ ac fields.

Here below, we describe the fabrication, magnetic properties, surface modification, and application of a single ferromagnetic layer, disk-shaped particles for targeted destruction of cancer cells in vitro.

\section{Size-dependent magnetic properties}

\subsection{Energy terms}

The magnetization distribution (spin arrangement) in small ferromagnetic particles depends on their size and can be non-uniform. It is well known that large particles, adopt multidomain states, where details of the magnetization distribution depend on the interplay between the exchange, magnetostatic and crystalline anisotropy energies (Fig. 1).

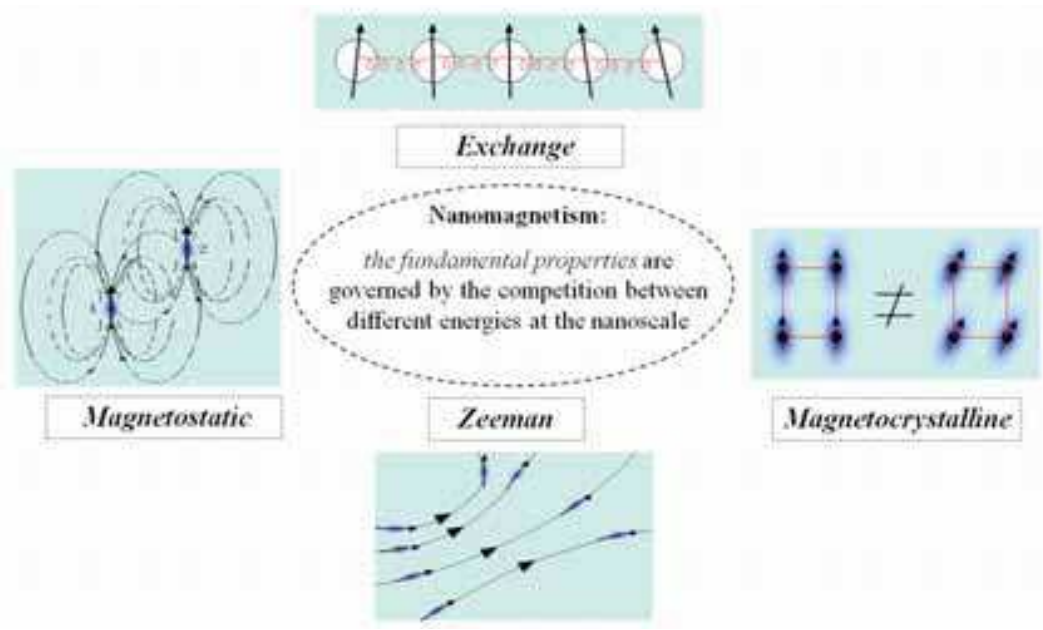

Fig. 1. Schematic representation of different energy terms governing the magnetic properties of small ferromagnetic elements. 
The short-range exchange interaction is responsible for parallel alignment of the neighboring spins. Although this interaction is a purely quantum mechanical phenomenon, it can be described phenomenologicaly, by introducing the material specific exchange constant. On the other hand, the magnetostatic interaction describes how the magnetic moments interact over long range distances within the ferromagnet.

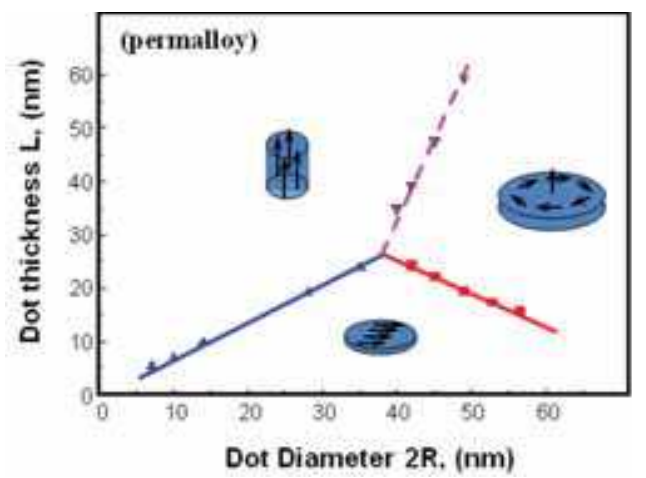

Fig. 2. Calculated magnetic phase diagram for disk-shaped Permalloy (Fe20Ni80) elements.

The magnetic vortex state is a ground state down to sub-100nm lateral dimensions of the disk.

The magnetostatic interaction energy is increased when the spin arrangement leads to formation of magnetic charges on the surface or within the sample volume. Shrinking the particle size leads to an increase of role of exchange energy; the magnetization distribution gradually becomes more uniform up to almost a saturated (in- or out- of plane single-domain states (Fig. 2). For immediate particle sizes and non-ellipsoidal particle shapes the spin structure becomes non-uniform with significantly reduced or even zero macroscopic moment in remanence. A model example is the "vortex" magnetization distribution in soft magnetic materials. Thus, it is the competition between the exchange and magnetostatic energies that leads to formation of domain walls or magnetic vortices. The size at which a particle becomes single domain depends on the balance between the above energies, on the shape of the particle and on its anisotropy.
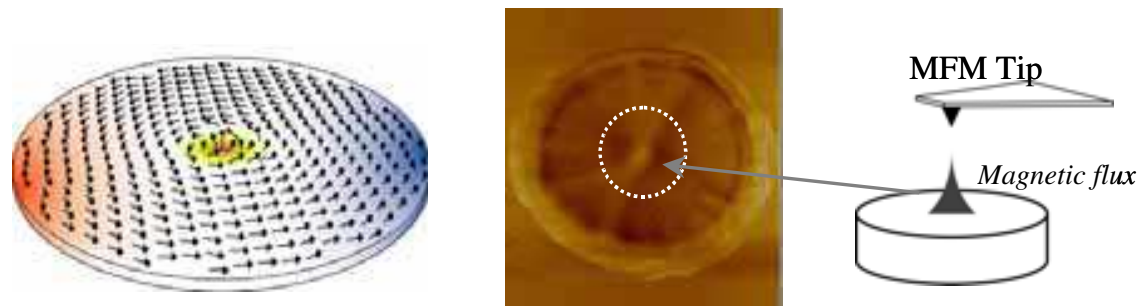

Fig. 3. Magnetization distribution in the magnetic vortex state obtained by micromagnetic modeling (left), and its magnetic force microscope image (right).

The characteristic magnetic length (so-called the magnetic exchange length) that results from the above energy competition is defined as $\xi_{\text {ex }}=\left(2 A \mu_{\mathrm{o}} / M_{\mathrm{s}}\right)^{1 / 2}$, where $A$ is the exchange constant and $M_{\mathrm{s}}$ is the saturation magnetization. A magnetically soft particle with lateral dimensions smaller than the exchange length is expected to remain in a single domain state at remanence. Here we are considering circular particles of permalloy ( $\mathrm{Py}, \mathrm{Fe}_{19} \mathrm{Ni}_{81}$ ) with 
thickness and diameter larger than $\xi_{\mathrm{ex}}$. It is well-established that a magnetic vortex structure is the ground state within a wide range of dot sizes from a few tens of $\mathrm{nm}$ up to few tens of microns (Scholz, et al., 2003; Chung, et al., 2010).

A magnetic vortex consists of a mainly in-plane flux-closure magnetization distribution with a central core that is magnetized perpendicular to the dot plane (Fig. 3). It was experimentally demonstrated by spin-polarized scanning tunneling microscopy that the core radius is about $10 \mathrm{~nm}$, i.e. approximately the material exchange length (Wachowiak, et al., 2002). A vortex can have a particular polarization $p= \pm 1$ that describes whether the core is oriented up or down and a particular chirality, i.e., the direction of the in-plane magnetization rotation.

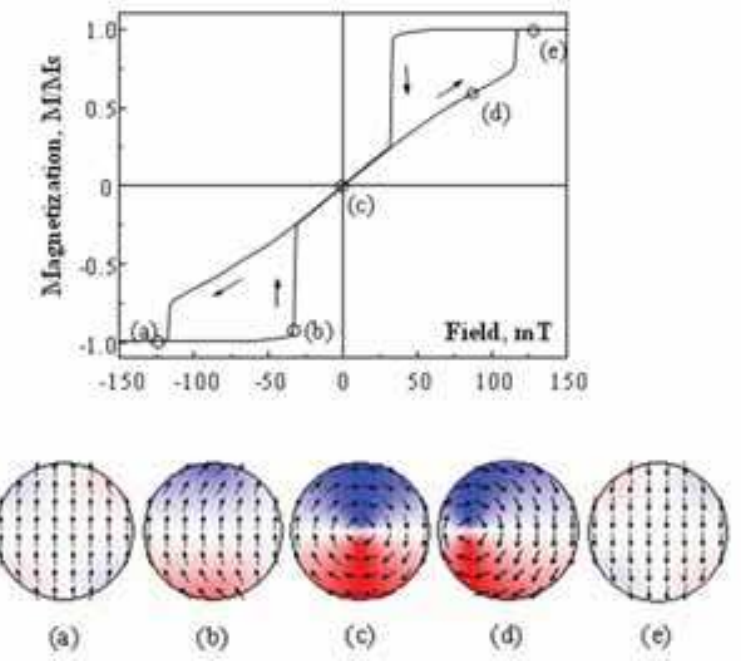

Fig. 4. The magnetization reversal in magnetically soft disk-shaped particle is accompanied by nucleation and annihilation of the magnetic vortex state.

The magnetization reversal process in individual particles can be effectively modeled using Landau-Lifshitz-Gilbert (LLG) micromagnetic calculations (Donahue, \& Porter, 1999). Figure 4 shows a typical hysteresis loop and the field evolution of the spin structure, calculated for $2 \mathrm{R}=200 \mathrm{~nm}$ diameter and $\mathrm{L}=30 \mathrm{~nm}$ thin circular dot. For the calculations, permalloy values for $M_{\mathrm{s}}\left(750 \mathrm{emu} / \mathrm{cm}^{3}\right)$ and $A\left(1.05 \times 10^{-6} \mathrm{erg} / \mathrm{cm}\right)$ were used and the magnetocrystalline anisotropy was neglected. The hysteresis loops were simulated by relaxing the system under sequentially applied magnetic fields to approximate a progression of equilibrium states, and simulations of the remanent state were carried out by allowing the system to relax from saturation in the absence of an applied field. The sample was modeled using three-dimensional $1 \times 1 \times 1 \mathrm{~nm}^{3}$ macrospin cells For all simulations a large damping parameter (0.5) was used. When the magnetic field is decreased from saturation (Fig. $4 a-4 b$ ), a magnetic vortex nucleates at the nucleation field $H_{n}$, accompanied by an abrupt decrease in the average dot magnetization. This results in a gain in the magnetostatic energy. The center of the vortex stays at the center of the dot at remanence (Fig. 3c). The reversible part of the loop corresponds to the vortex core movement perpendicular to the applied field (Fig. 3d). When the magnetic field reaches the annihilation field $H_{\text {an }}$ the vortex vanishes completely. This process stabilizes the saturated, single-domain state in the dot (Fig. 3e). The values of $H_{n}, H_{\text {an }}$, and the slope of the linear part of the hysteresis loop are strongly size dependent (Guslienko, et al., 2001). 

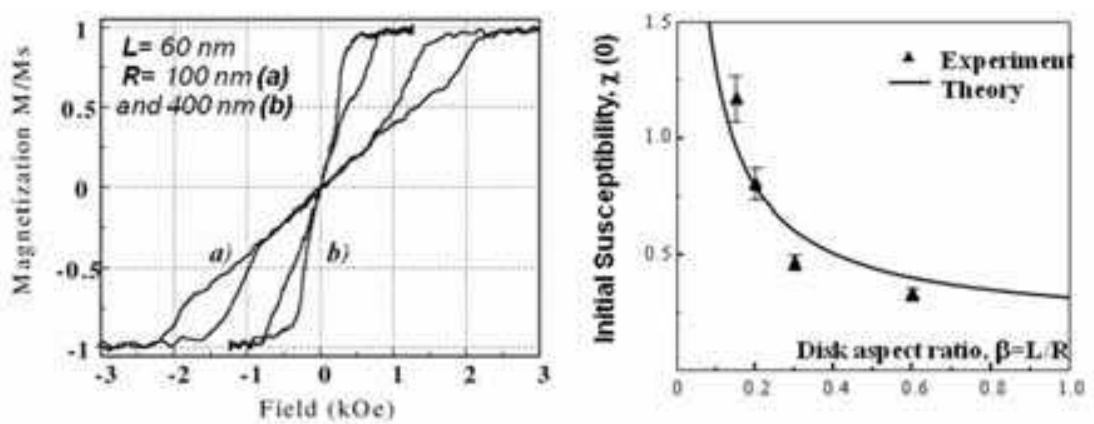

Fig. 5. Size dependent experimental hysteresis loops (left) and susceptibility (right) for submicron permalloy disks.

For example, Fig. 5 (left plot) compares the experimental hysteresis loops measured for two kinds of dot arrays with the same thickness and different diameters of 200 and $800 \mathrm{~nm}$. Decrease in the dot aspect ratio leads to reduced vortex stability, and hence to decrease in the vortex nucleation and annihilation fields. Similarly, the magnetic susceptibility (Fig. 5, right plot) scales as a function of the dot aspect ratio for given materials. This suggests that the magnetic properties of the soft (with no crystalline anisotropic) magnetic dots are entirely predetermined by the geometry of the sample, e.g. precisely controlled lateral dimensions and thickness during the fabrication process will result in magnetically undistinguishable particles.

\subsection{Spin dynamics}

Besides unique reversal process, the vortex magnetization distribution leads to a considerable modification of the spin excitation spectrum in comparison to those in the uniform (single domain) state. The dynamic response of the vortex core can be considered as a harmonic oscillator trapped in parabolic potential well due to magnetostatic interaction (Fig. 6, left plot). Thus, when the sample is subjected to a time-varying (ac or pulse-like) magnetic field, the vortex core experiences a force (gyroforce) perpendicular to its displacement from equilibrium position that results in a relatively slow (sub-GHz) frequency, spiral-like motion of the core. This excitation is known as a translational or gyrotropic mode of the magnetic vortex. The direction of rotation depends only on the core polarization, as demonstrated in time-resolved magneto-optical Kerr (Park, et al., 2003) and X-ray magnetic circular dichroism imaging experiments (Ade, \& Stoll, 2009). The other higher frequency (sub-ten $\mathrm{GHz}$ ) excitations within some approximation can be understood as the quantized spin waves in restricted geometries (Demokritov, et al., 2001).

Surprisingly, the summarized results of the calculations for different dot thickness $L$ and diameter $2 R$ scales universally when plotted as a function of the disk aspect-ratio $L / R$. (Fig. 6 , right plot). The eigenfrequency of the vortex translational mode in a disk-shape dot can calculated analithycally (Guslienko 2001) as:

$$
\omega_{0}=2 \gamma M_{s}\left[4 \pi \int_{0}^{\infty} d t t^{-1} f(\beta t) I^{2}(t)-0.5\left(\xi_{e x} / R\right)^{2}\right],
$$

where $\gamma$ is the gyromagnetyic constant, $\beta=L / R, f(x)=1-(1-\exp (-x)) \not x$ and $\xi_{\mathrm{ex}}$ is the magnetic exchange length. Therefore, the frequency can be predicted and adjusted if needed just by 
modifying $R$ or $L$. Please note, that the value of all frequencies is in $\sim 100 \mathrm{~s} \mathrm{MHz}$, accessible by using rather conventional instrumentation. The resonant properties of ferromagnetic microdisks may offer an additional and very important functionality for bio-medical applications. Namely, the disk-shaped particles subjected to ac magnetic field oscillating at the frequency of the vortex translational mode will demonstrate strong enhancement of the absorption losses. Therefore, it could be possible to significantly improve the magnetic particles detection using experimental set-up schematically shown in Fig. 7. The magnitude of real and imaginary part of the dynamic permeability could be used for counting the magnetic particles flowing through the measurements cell. A significant increase in absorption losses (and therefore, enhanced sensitivity) is expected at the frequencies when the eigenmodes of collective spin motion are excited. In principle, one should be able to achieve a single particle detection via the dynamic susceptibility measurements.
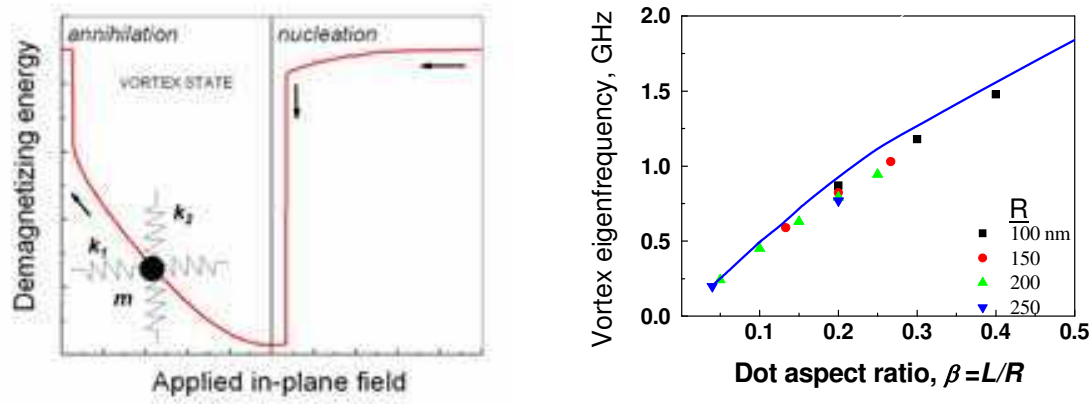

Fig. 6. The magnetic vortex core shows a harmonic oscillator response and can be viewed as a particle trapped in a potential well (left plot). The eigenfrequency of the system scales universally as a function of the disk thickness to diameter ratio (right plot) and can be finetuned in sub-GHz frequency range.

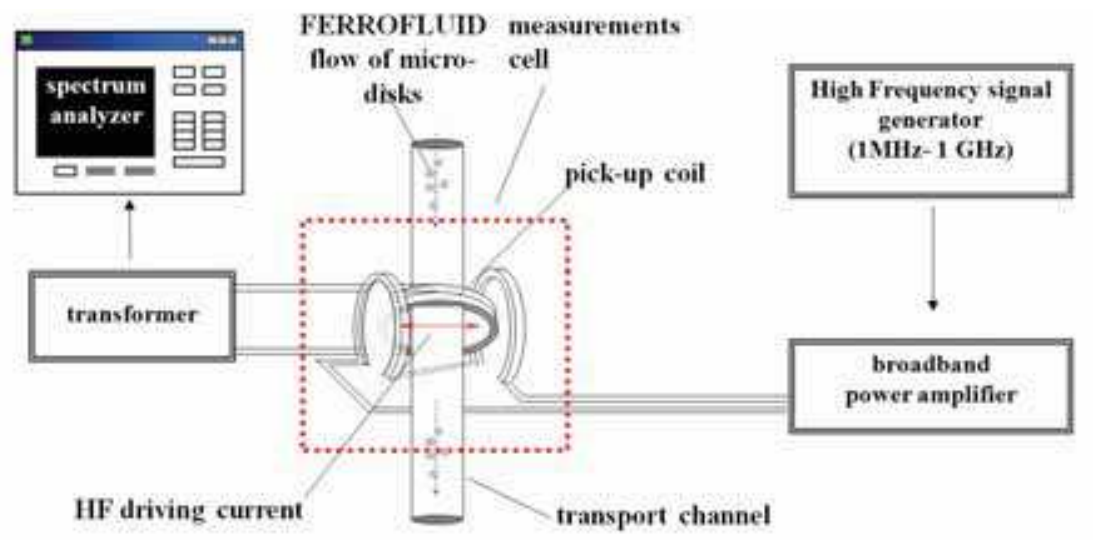

Fig. 7. Block-diagram of the experimental set-up for detection of magnetic particles (highfrequency permeability measurements). The spectrum analyzer attached to a pickup coil; a separate drive coil is connected a 1 watt, up to $1 \mathrm{GHz}$, broadband amplifier driven by a frequency generator. 


\subsection{Magnetostatic interaction}

Since we are considering use of fluid suspension of the particles, the inter-particle interaction effects should be carefully examined.
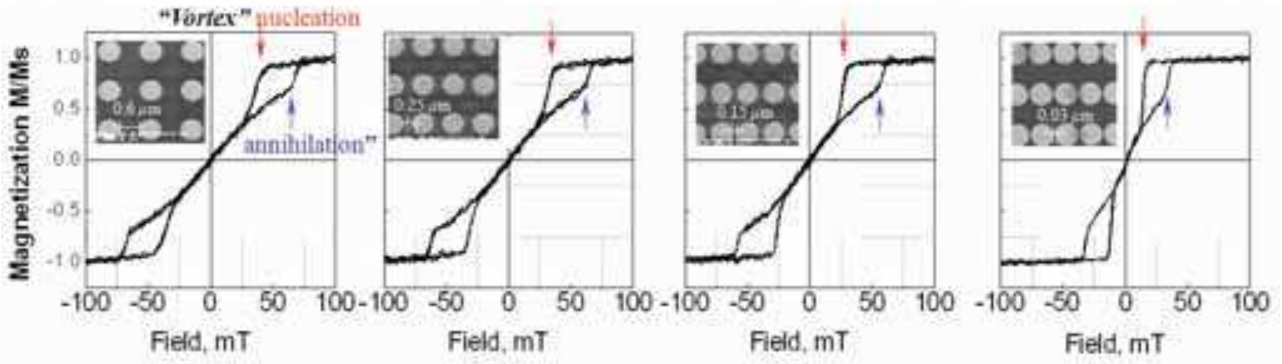

Fig. 8. The interdot magnetostatic interaction decrease the spin vortex state stability, yet, even for very small separation distances the particles show zero net magnetic moment in remanence due to vortex state formation. The later eliminates the long-range magnetostatic forces otherwise responsible for magnetic particle agglomeration.

Figure 8 compares four hysteresis loops for 40nm thin dots with a diameter of $600 \mathrm{~nm}$, but variable interdot distances ranging from 600 to $30 \mathrm{~nm}$. The field was applied along the horizontal direction. Macroscopic measurements technique yields information on the magnetization reversal process averaged over many 1000's of dots. Nevertheless, the loops have a clear signature of the magnetization reversal with "nucleation" and "annihilation" of magnetic vortices. We find that hysteresis loop depend not only on the dot diameter and the thickness, but also on the interdot distance. As seen in Fig. 8, nucleation and annihilation fields decrease, whereas an initial susceptibility of the vortex increases with decreasing interdot distance. Although the dipole-dipole interaction decreases the vortex state stability for small inter-particle separation distances, it is possible to design the particles so that they posses vortex ground state even for smallest separation distances (Novosad, et al., 2002). This is important since magnetic flux closure reduces the long-range magnetostatic forces otherwise responsible for magnetic particle agglomeration.

\section{Magnetic vortex microdisks for targeted cancer cell destruction}

So far we were considering properties of the individual disks arranged in planar arrays on the wafer. By properly modifying the microfabrication process it is possible to release the disks into solution.

\subsection{Microfabrication}

The fabrication process includes optical lithography and magnetron sputtering (Kim, et al., 2010). Use of the master mask with $1 \mu \mathrm{m}$ diameter disks arranged on a $3 \times 3 \mu \mathrm{m}^{2}$ square lattice covering $3 \times 3 \mathrm{~cm}^{2}$ area will result in $\sim 10^{8}$ disks.

The process starts with negative tone photoresist spin coating on a silicon wafer. After the master mask is placed in contact with the layer of pre-baked photoresist, the sample is illuminated with UV light. An organic solvent dissolves and removes photoresist that is not exposed. Finally, magnetron sputtering is used to deposit a $5 \mathrm{~nm}$ underlayer gold, followed by $60 \mathrm{~nm}$ of permalloy, and topped with another $5 \mathrm{~nm}$ of gold layer. The disks can easily be 


\section{Negative Tone Photoresist Spin-coating}

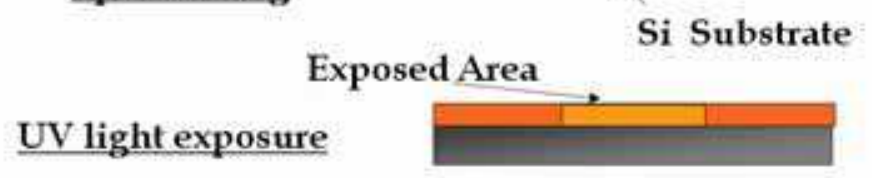

\section{Resist Development \\ Thin Film Deposition (FeNi target)}

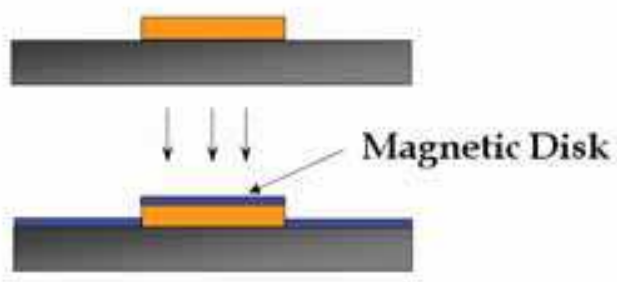

Fig. 9. Microdisks fabrication of MDs by optical lithography and magnetron sputtering.

released from the wafer by the lift-off process in acetone. The approach allows low cost production of uniformly sized particles (Fig 10). The details of the process flow schematically shown in Fig. 9 are given below.

- Resist: N-1410 negative tone resist supplied by Micro Resist Technology GmbH (www.microresist.de).

- Optical lithography mask: 1 micron diameter circular dot array arranged in 3 micron period square lattice covering a $3 \times 3 \mathrm{~cm}^{2}$ area was used.

- Wafers: 2 inches prime quality (100) silicon wafers with surface flatness $<2 \mu \mathrm{m}$ supplied by University Wafers Inc., (www.UniversityWafer.com)

- Resist spin coating: Speed $3000 \mathrm{rpm}, 60 \mathrm{sec}$; resulting resist thickness of $\sim 1$ micrometer.

- Hot plate baking: $90^{\circ} \mathrm{C}$ for 120 sec.

- Exposure: $365 \mathrm{~nm}$ light; $150 \mathrm{uC} / \mathrm{cm} 2$ for $10 \mathrm{sec}$.

- Resist Development: 533-S solvent, 30-40sec.

- 5 nm Au/ 60nm Fe20Ni80/ 5nm Au deposition: DC magnetron sputtering (power 50W), base pressure $5 \times 10^{-8}$ Torr; deposition rate 1A/sec @ 3 mTorr Ar pressure.

- Lift-off process: acetone, $30 \mathrm{~min}$, ultrasound assisted.

- Centrifugation: in 3 steps, 10 min @ 3,500 rpm, with fresh acetone wash between steps.

- Transfer into aqueous solution: $24 \mathrm{~h}$ dialyze in MilliQ followed by centrifugation and dispersion in phosphate buffer solution (PBS, $0.1 \mathrm{M}, \mathrm{pH}$ 7.2) for further biofunctionalization.

Fig. 10 shows the representative optical microscope image of the dried suspension of the as fabricated microdisks.

Here we focus on testing $\sim 1$ micron diameter disks. It migh be desirable, especially from the view-point of future in-vivo application, to scale the system down to $100 \mathrm{~nm}$ dimensions. In this regard, the following shold be concidered: 1) Scalability of the spin vortex state. The Fig. 2. shows theoretical magnetic phase diagram of the magnetically soft disks shape particle. The magnetic vortices in deep submicron disks were experimentally imaged by many groups, see for example (Chung, et al., 2010). Thus, from the energy minimization viewpoint the magnetic vortex state remains stable ("ground") spin state for magnetically 


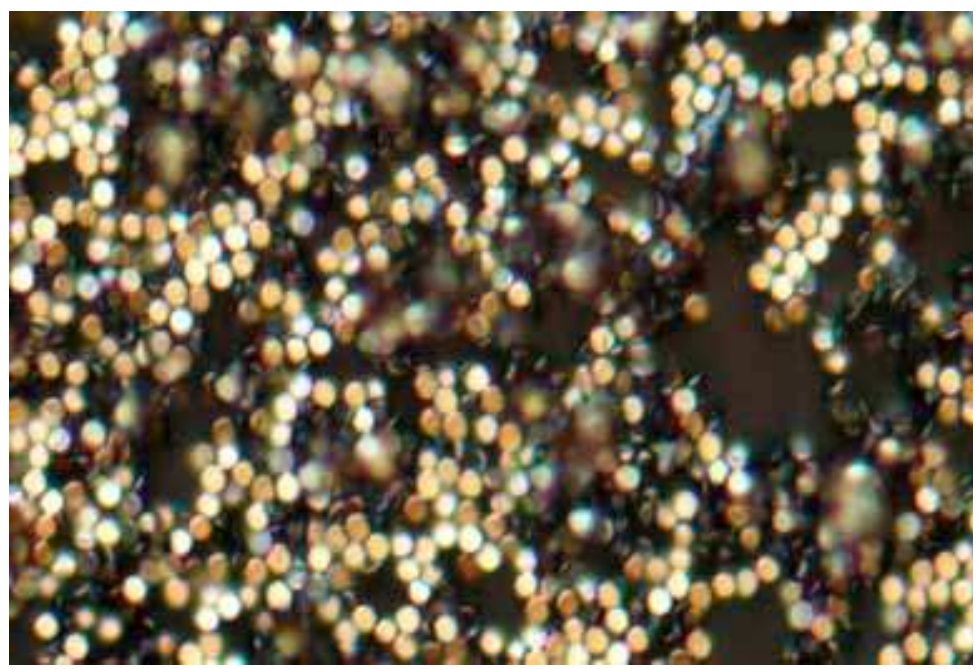

Fig. 10. A high resolution optical microscope image of the dried suspension of 60 -nm-thick, 1 micron diameter permalloy disks coated with a 5-nm-thick layer of gold on each side. The discs were prepared by means of magnetron sputtering and optical lithography (Fig.9).

soft disk down to deep sub-100 nm diameters. 2) Feasibility of nanofabrication. Mass production of $\sim 100 \mathrm{~s} \mathrm{~nm}$ diameter disks can be achieved using more advanced technique, such as nanoimprint lithography. The disks detachment from substrate (after gold / magnetic / gold films deposition) can be facilitated by employing bi-layer polymer resist structure combined with ultrasonic-assisted lift-off process. Importantly, nanoimprint lithography is based on "stamp \& repeat" approach, e.g. with using a master mask that can be employed many times. It is a truly a low cost and mass production method with exceptionally narrow size distribution of the final structures ( $\mathrm{Hu}$, et al., 2008, Wang, et al., 2008). 3) Scaling of the magneto-mechanical coupling strength. Decrease in the disk size will inevitable decrease the total magnetic moment $\mathbf{m}$ per disk (because of reduced particle volume). One can expect that the magnetic torque $\mathbf{m} \times \mathbf{H}$, where $\mathrm{H}$ is applied magnetic field, will decrease accordingly. However, this torque that drives rotational motion of the nanodisk can easily be recovered by increasing the magnetic field amplitude. For instance, ten times smaller volume disks (e.g. using $\sim 270 \mathrm{~nm}$ diameter, $\sim 17 \mathrm{~nm}$ thin disks instead of the currently used ones), will experience the same magnetic torque if subjected to $\sim 2.7 \mathrm{kOe}$ field, instead of $\sim 90$ Oe field used in the in-vitro experiments below. A stronger magnetic fields are easy to achieve since the required frequency of magnetic field is of few $\sim 10 \mathrm{~s} \mathrm{~Hz}$ only. Thus, there appear no major conceptual or feasibility problems to employ the nanosize magnetic disks.

\subsection{Magnetic properties}

After fabrication the samples were characterized using conventional magnetometry and light scattering experiments. The hysteresis loop data (not shown) agree with the concept of the magnetization reversal due to nucleation and annihilation of the magnetic vortices. In zero field (remanent state) the vortex core is in the center of each disk, whereas under 
magnetic field application the vortex shifts (reversibly) to develop the magnetization component parallel to the field (see Fig. $4 \mathrm{~d}$ ). Owning to the flat geometry, the disks rotate under an alternating magnetic field. This can be used for testing the concept of advanced therapies, which include combined drug delivery and magneto-mechanical cell destruction when targeting tumor cells. To verify a mechanical responsiveness of the microdisks we investigated the field-dependent intensity of laser beam passing though the aqueous solution $\left(10^{7}\right.$ disk / ml), Fig. 11 (left). In the absence of the magnetic field, the disks are randomly oriented causing significant light scattering. Under application of magnetic field parallel to the laser beam direction, the disks are instantly aligned to have their plane parallel to the field. The scattering cross-section is therefore reduced leading to a rapid increase in the transmitted light intensity (Fig. 11, right).
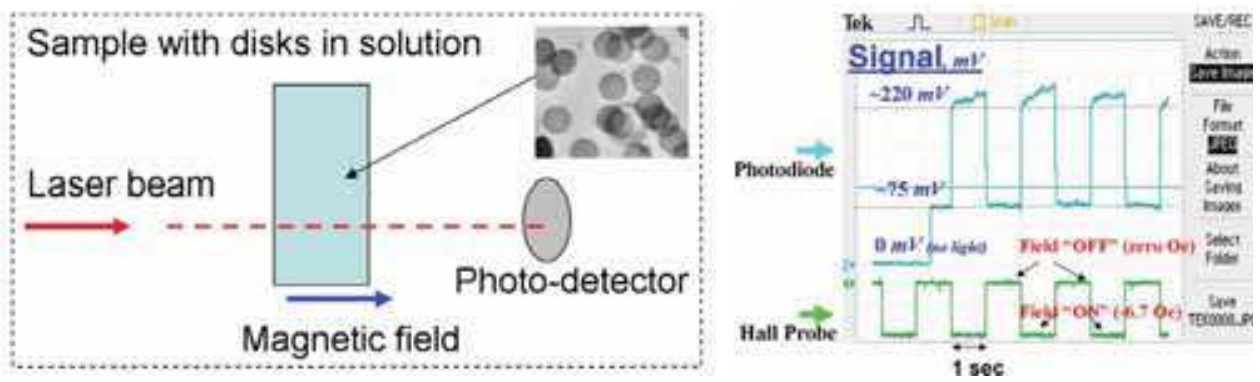

Fig. 11. Schematics of the experimental arrangement to probe the microdisk responsiveness to an external magnetic field (left) and representative data for $7 \mathrm{Oe} / 1 \mathrm{~Hz}$ applied field (right). The light scattering cross-section is reduced leading to a rapid increase in the transmitted light intensity.

Consequence of the magneto-mechanical coupling is that the magnetic vortices can be used as a mediators to deliver the energy of an external source (of magnetic field) to the cell. Thus, the targeted cell destruction for cancer therapy becomes possible.

\subsection{In vitro experiments}

The feasibility of the proposed concept was tested using Glioblastoma multiforme (GBM), an aggressive form of brain cancer a model for an in vitro experiment (Kim et al., 2010). The specific targeting of microdisks to N10 glioma cancer cells (Da, at al., 1990) was achieved by functionalization of the inorganic material with antihuman-IL13 $\alpha 2 \mathrm{R}$ antibody (hereafter referred to as $\mathrm{mAb}$ ), because IL13 $\alpha 2 \mathrm{R}$ is over-expressed on the surface of glioma cells (Debinski, et al., 1999; Kawakami, at al., 2001) and serves as a marker for targeting by cytotoxic elements, including nanoparticles (Rozhkova, et al., 2009). Thiolate groups that readily react with the gold surface were first randomly introduced into the mAb using Nsuccinimidyl-S-acetylthioacetate (SATA). After deprotection of the thioacetylated protein, the free thiolate groups of the mAb immediately reacted with the gold surface of the microdisks (Fig. 12).

To evaluate the magnetic field-induced cytotoxicity the loss of cell membrane integrity was assayed via release of cellular lactate dehydrogenase (LDH test). The N10 GBM cells were incubated with the mAb-modified microdisks and exposed to an ac magnetic field of $90 \mathrm{Oe}$ with frequency of $20 \mathrm{~Hz}$ using experimental set-up shown in Fig. 13. 


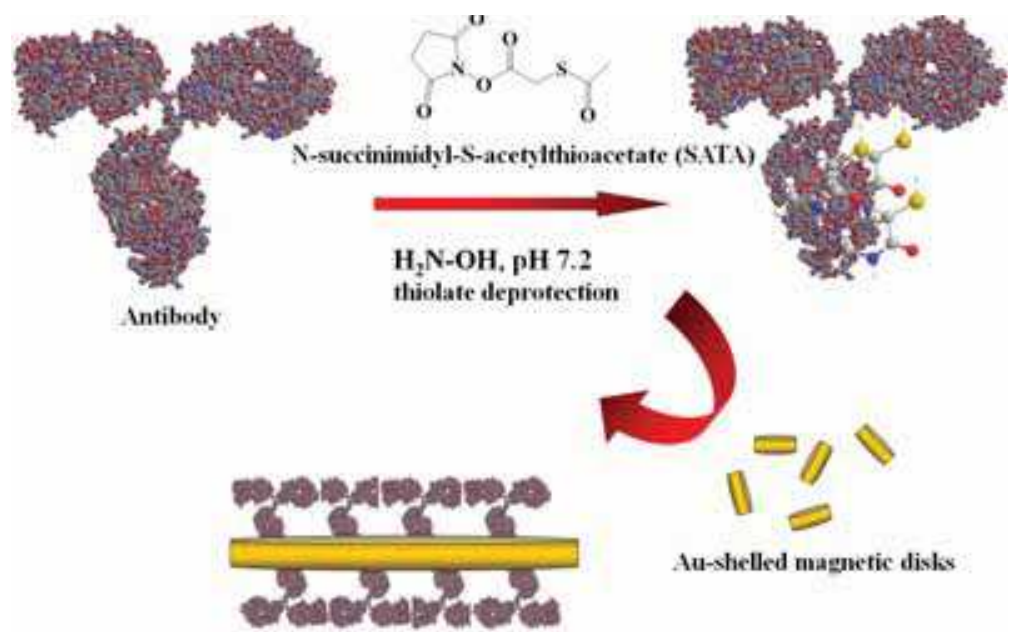

Biofunctionalized magnetic disks

Fig. 12. The microdisk biofunctionalization with antihuman-IL13 $\alpha 2 \mathrm{R}$ antibody.

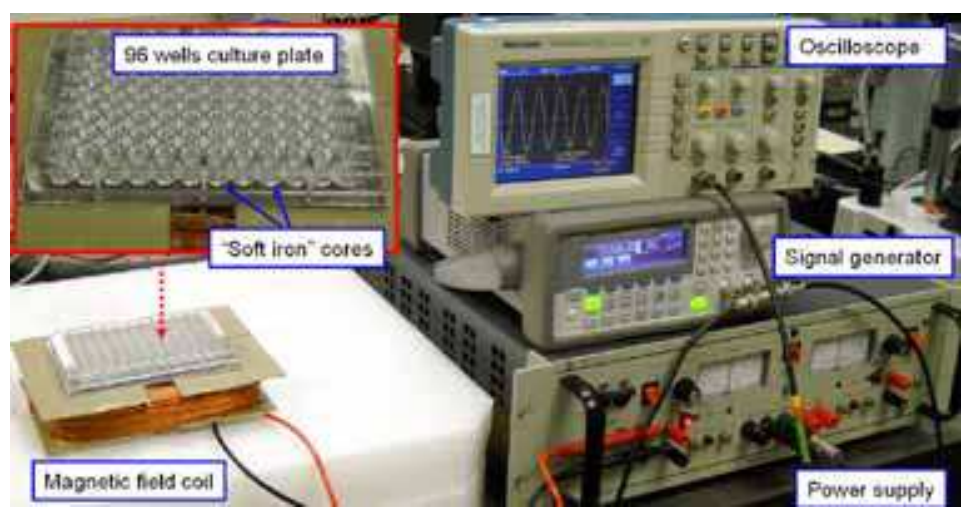

Fig. 13. The experimental set-up for magnetic field application in-vitro.

Field-treated MD-mAb-cell culture exhibited up to $\sim 90 \%$ cytotoxicity effect (Kim et al., 2010). Furthermore, dramatic cell morphology changes were observed after the magnetic field application (Fig. 14). Figure 15 compares representative AFM images of the control N10 cells pre-incubated with MD-mAb (left), and the cells after magnetic field exposure (right) after $1 \mathrm{hr}$ recovery culture. Since the observed morphological characteristics are typical for apoptosis, we also demonstrated other important characteristics of apoptotis, - severe nuclear DNA nicking (data not shown). Apoptosis is one of the main types of programmed cell death used by nature for elimination of damaged or unneeded cells without local inflammation (Mpoke, \& Wolfe, 1997). The fundamental importance of correct execution of apoptosis is evident from the many human diseases with aberrancies in apoptosis, including cancer. During cancer development, various imbalances can arise in the apoptotic machinery (Kasibhatla, \& Tseng, 2003). 

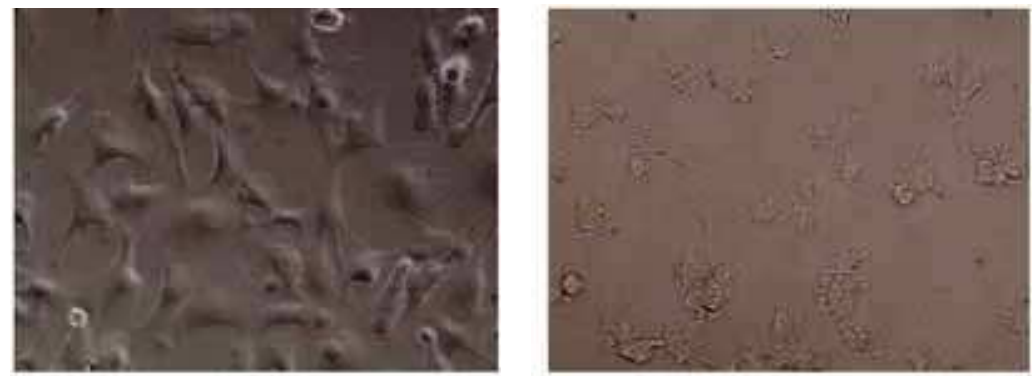

Fig. 14. Representative $\mathrm{x} 50$ optical microscope images of cancer cells for two distinctive cases. Left image: Binding experiments: Human glioma N10 cells incubated with IL13functionalized disks; no field was applied. The morphology of the cell is essentially the same as for control sample (e.g. cells without magnetic disks; not shown). Right image: an optical micrograph of the cells incubated with IL13-functionalized disks after $20 \mathrm{~Hz} / 90 \mathrm{Oe} a c$ magnetic field was applied for $10 \mathrm{~min}$.
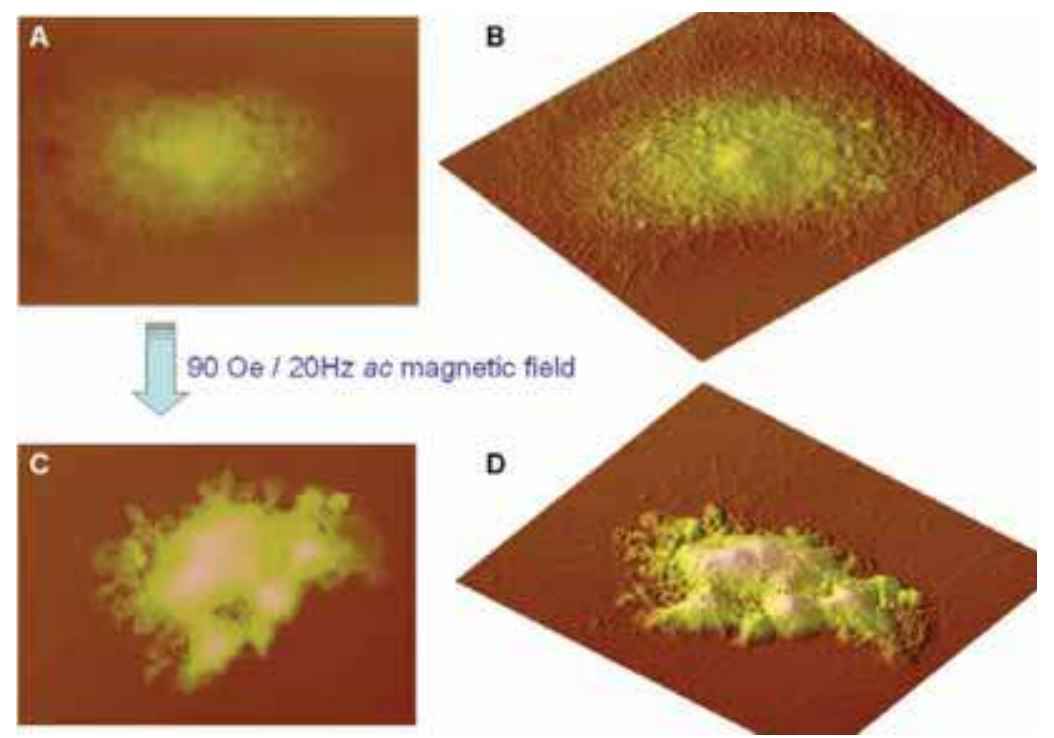

Fig. 15. 2D and 3D Atomic Force Microscope images of control (conjugated with MDs, but untreated) cell (upper images), and treated N10 glioma cell subjected to $90 \mathrm{Oe} / 20 \mathrm{~Hz}$ magnetic field (lower images).

Consequently, sensitivity towards apoptosis is progressively reduced, which ultimately leads to inappropriate cell survival, resistance to cytotoxic drugs, and malignant progression. Induction of apoptosis plays a central role as a potential anticancer strategy (Kasibhatla, \& Tseng, 2003). Although a detailed understanding of the intracellular pathways responsible for cell death induced by magneto-mechanical actuation is still lacking, the proposed approach opens wide research horisonts to explore applicability of magnetic particles for anti-cancer theraphies using low frequency magnetic fields. 


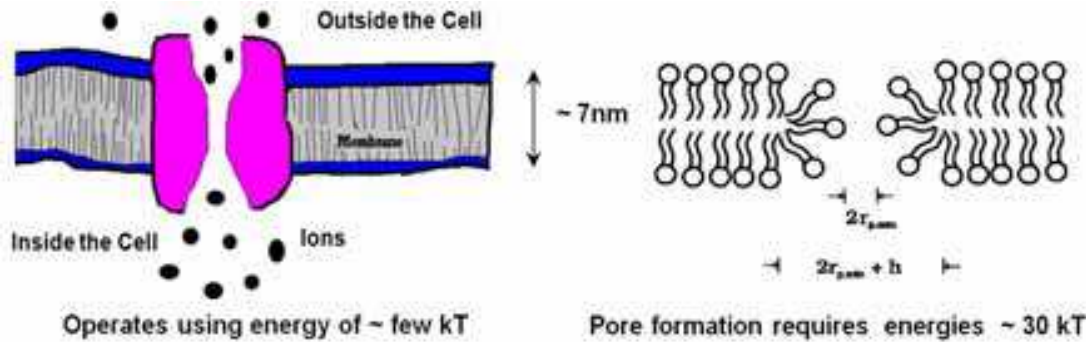

Fig. 16. The magnetic vortex forces are strong enough to mechanically actuate the existing ion channels (left), as well as to interrupt the barrier function of the cell membrane through formation of $\sim 10 \mathrm{~nm}$ pores (right). The later could lead to altered molecular transport into and out of cells, therefore altering intra cellular machinery function.

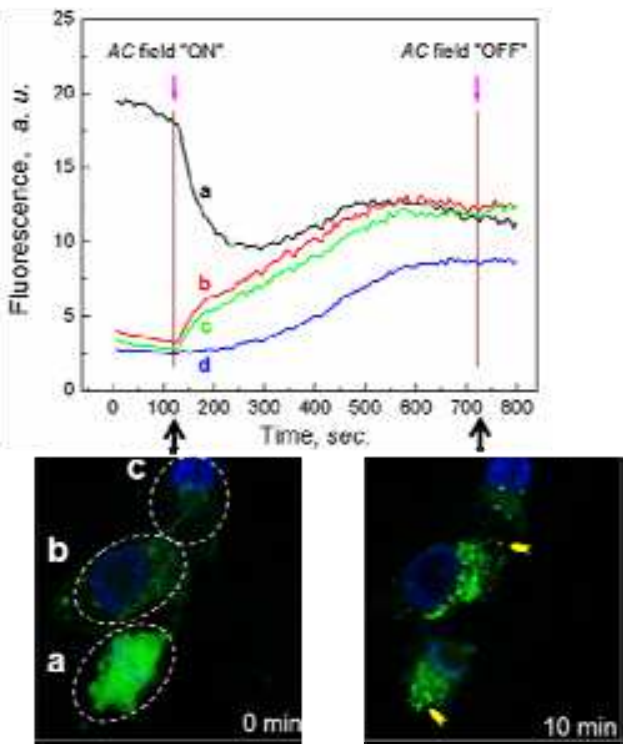

Fig. 17. Time-evolution of the calcium fluorescence signal averaged for three difference cells ( $a, b$ and $c$ ), as well as for whole field of microscope view (d), and Optical microscope image calcium ffluorescence intensity image prior (left) and after (right) application of $a c$ magnetic field.

In these experiments, the magnetically actuated microdisks delivered to the cell surface mechanical force of $\sim 50-100 \mathrm{pN}$ (upper limit estimate). On the other hand, forces as small as few $\mathrm{pN}$ were reported to be sufficient to activate a single ion channel (Guharay, \& Sachs, 1984; Martinac, 2004; Mannix, 2007). Furthermore, a theoretically calculated energy barrier (Vaughan, \& Weaver, 1996) to create a pore in the cell membrane comparable in diameter to its thickness $(\sim 7 \mathrm{~nm})$ using rotational forces produced by a magnetic particle is of order $\sim 30$ $\mathrm{k}_{\mathrm{B}} \mathrm{T}$, where $\mathrm{k}_{\mathrm{B}}=1.38^{*} 10^{-23} \mathrm{~J} / \mathrm{K}$ is the Boltzmann constant, $\mathrm{T}$ is the temperature. For comparison, the Zeeman energy of the magnetic vortex subjected to 100 Oe field is of $\sim 1^{*} 10^{17}$ $\mathrm{J}$, e.g. of order $\sim 1000 \mathrm{k}_{\mathrm{B}} \mathrm{T}$. Thus, the magnetic vortex forces are strong enough to 
mechanically actuate the existing ion channels, as well as to interrupt the barrier function of the cell membrane through pores formation. The later could lead to altered molecular transport into and out of cells, therefore altering intra cellular machinery function. To clarify this possibility, we have performed experiments on imaging of intercellular calcium relocalization during application of ac magnetic field $(10 \mathrm{~Hz} / 90 \mathrm{Oe})$ in $N 10$ glioblastoma cells. The role of the calcium as a universal secondary messenger in execution of the cell-death process is well-established and is reported in connection with the studies of drug-induced cytotoxicity (Clapham, 1995; Duchen, 2000; Boehning, et al., 2003; Mattson et al., 2003; Smulson, et al., 1999; Yakovlev, et al., 1999).

To investigate whether calcium ions signaling is involved in the observed magnetic-vortexdriven cell death the N10 cells were preloaded with Fluo-4AM, a calcium indicator dye and studied using a dual wavelength laser confocal microscopy. A magnetic coil was mounted on the microscope sample stage. Figure 17 shows semi-quantitative data on changes in intracellular concentration of calcium during ac field application in a nominally calcium-free Hank's buffer solution. Fluorescence intensity was detected in areas $\mathbf{a}, \mathbf{b}, \mathbf{c}$ corresponding to three cells with different initial fluorescence intensities due to different steps of a life cycle. Total fluorescence (including cells and background) was measured as an area d. Once ac field is applied noticeable changes in fluorescence were detected. Thus, for cells $\mathbf{b}$ and $\mathbf{c}$ constant steady increasing of fluorescence intensity (up to two-folds) was observed. In contrast, for the cell $\mathbf{a}$ with originally higher calcium concentration initial sharp slope was followed by smooth increase of calcium levels.

The global increase in the cytoplasmic calcium triggers the simultaneous release of cytochrome $c$ from mitochondria, formation of the apoptosome and activation of specific protease caspases. Caspases together with nucleases then finalize the cell death process by cleaving protein substrates and nuclear DNA. Importantly, the number of TUNEL-stained cells was observed to fall remarkably ( $50 \%)$ after the addition of $20 \mu \mathrm{M} \mathrm{Z-VAD-FMK,} \mathrm{an}$ inhibitor of caspase proteases, the key role-players in apoptosis. Therefore, we can anticipate that magnetic vortex mediated mechanical stimulus may eventually be converted into a downstream chemical signal cascade able to initiate apoptosis and cell destruction.

Altogether these confocal microscopy data confirmed our proposal on magnetomechanically-induced ionic channels actuation or/and cell membrane pores formation altering calcium homeostasis and resulting in re-programming of important intracellular biochemical pathways. Future studies, including application of specific inhibitors of stretchactivated membrane protein complexes and experiments with controlled concentrations of calcium in cell culture media may help to further clarify intriguing details of the proposed mechanism. The details of the above described in vitro experiments and used materials are given below.

\section{Materials \& methods}

Materials: Monoclonal anti-human-IL13Ralpha2 (designated mAb), cat\# MAB6141, clone 83807, human IL-13 Ra2 biotinylated affinity purified polyclonal detection antibody, cat \# BAF614, and Mouse IgG1 Isotype Control, cat \# MAB002, clone 11711, were from R\&D Systems, Minneapolis, MN, USA. N-Succinimidyl S-Acetylthioaceteate (SATA), dimethylsulfoxide (DMSO), hydroxylamine $\mathrm{HCl}$, Ethylenediaminetetraacetic acid tetrasodium salt dihydrate (EDTA) were from Sigma (St. Louis, MO, USA). Poly-L-lysine $(0.01 \%$ soln) was purchased from Sigma (St. Louis, USA). Other reagents of the highest 
grade available were obtained from Sigma IR spectra were recorded with a FT-IR spectrophotometer (Bruker Vertex 70, Billerica, MA). For cell imaging Axio imager Z1m; camera: Axio Cam MRM; image analysis program: Axiovision were used. Atomic Force Microscope was from Nano V, Veeco (Santa Barbara, CA, USA). CytoTox-ONE Homogeneous Membrane Integrity Assay kit (Promega, Madison, WI, USA). ApopTag®.

Plus Peroxidase In Situ Apoptosis Kit was from Chemicon (Millipore). Z-VAD-FMK (carbobenzoxy-valyl-alanyl-aspartyl-[O-methyl]-fluoro-methylketone) caspase inhibitor was purchased from Promega Corp.

Cell culture. The human glioma tumor cell line No. 10 cells (N10) were purchased from the Japanese Tissue Tumor Bank (Tokyo, Japan). No. 10 cells were grown in 96-well plates in RPMI medium (Gibco, Life Technologies, USA) supplemented with 10\% (v/v) FBS (CellGrow, MediaTech, Heydon, VA), $2 \mathrm{mM}$ L-glutamine, and penicillin/streptomycin, and incubated in a humidified atmosphere with $5 \% \mathrm{CO}_{2}$ at $37{ }^{\circ} \mathrm{C}$.

Bio-functionalization. $27.8 \mu \mathrm{l}$ of $55 \mathrm{mM}$ SATA solution in DMSO and $2.8 \mu \mathrm{l}$ of $60 \mu \mathrm{M}$ IL13 solution were mixed and incubated at room temperature with continuous gentle shaking in the dark for 30 minutes. To deprotect thiolate groups the SATA-modified antibody solution was combined with $4 \mu \mathrm{l}$ of $0.5 \mathrm{M}$ hydroxylamine $\mathrm{HCl}$ and $25 \mathrm{mM}$ EDTA solution in $0.1 \mathrm{M}$ PBS, pH 7.4 and incubated for 2 hours at room temperature. $500 \mu$ l of MDs suspension $\left(5 \times 10^{8} \mathrm{ea} / \mathrm{ml}\right)$ were degassed by nitrogen gas bubbling for 15 minutes, and then the deacetylated antibody solution was immediately mixed with the MDs suspension with continuous gentle shaking in the dark at room temperature for $\sim 2$ hours. The final MD-mAb was spin-washed 3 times with $500 \mu \mathrm{L} 0.1 \mathrm{M}$ PBS, pH 7.4 to remove unbound protein, and then redispersed in $500 \mu \mathrm{l}$ of $0.1 \mathrm{M}$ PBS, pH 7.4 and stored at $4 \mathrm{C}$.

Magnetic field application. A magnetic field station plate was made by inserting $1 / 4$ inch soft iron rod into the under well in 96 well-plate. The station circumference was wound by $\mathrm{Cu}$ wire as a coil. Power supply/amplifier (KEPCO, Flushing, NY, USA), Function generator (Agilant 33220A, USA), oscilloscope (TEKTRONIX, Beverton, OR, USA) was connected with the magnetic field station for generating ac magnetic field. For experiment 96 well plate with samples was positioned on to of magnetic field station plate. Magnetic field strength was calibrated with Hall probe. After the treatment, the necrosis and apoptosis of treated cells was assessed with standard lactate dehydrogenase (LDH) test, Terminal Deoxynucleotidyl Transferase dUTP nick end labeling (TUNEL) assay.

Morphological analysis of cell viability by optical and atomic force microscopy. Human glioma cells from the N10 cell line were cultivated on poly-L-lysine coated coverslips. The human glioma tumor cell line No. 10 cells (N10) were grown on the coverslips in 24 well plates for 24 hours to reach $10^{4}$ cells per a coverslip. The cells on the coverslips were exposed to ac magnetic fields as it is described above in the magneto-mechanical cell destruction setup. The treated cells were fixed in $4 \%$ paraformaldehyde and analyzed by optical microscopy or recovery cultured in standard conditions for 4 hours and then fixed for AFM. Coverslips were thoroughly rinsed with MilliQ water prior to imaging. Bright or dark field images of the cells were taken using a 10X, 20X, 50X and 100X of objective lens. AFM surface images of the cells were acquired in non-contact mode.

Laser confocal intracellular calcium imaging. For the Calcium assays the N10 cells were grown in $35 \mathrm{~mm}$ Fluorodish cell culture dishes (World Precision Instruments, FL, USA) to reach $\sim 10,000$ cells per dish and then incubated with MD-mAb at standard conditions. Cells were loaded with $1 \mu \mathrm{M}$ Fluo-4-AM containing 0.02\% Pluronic ${ }^{\circledR}$ F-127 (Molecular Probes) 
and $1 \mu \mathrm{g} / \mathrm{ml}$ Hoechst 33342 (Molecular Probes) at $37{ }^{\circ} \mathrm{C}$ for 20 and $5 \mathrm{~min}$ respectively, washed and then assayed in Hank's buffer. A magnetic coil was placed on top of a sample stage of a dual-wavelength Leica SP5 Tandem Scanner Spectral 2-Photon Confocal Microscope. After 2 min of dye-loaded cell initial fluorescence imaging, an alternating magnetic field was applied to stimulate MDs on the cell surface during $10 \mathrm{~min}$.

ELISA. The human malignant glioma N10 cell line was grown in 96 well plate for 24 hours to reach $10^{4}$ cells per well. Next day cells were blocked with PBS supplemented with $4 \%$ BSA for 1 hour at 37C followed by 3 times PBS washing. MD-mAb or free antibodies in concentrations $1-105 \mathrm{ng} / \mathrm{ml}$ were added to the cells. After incubation with conjugates or free antibodies cells were washed and incubated with secondary anti-mouse HRP conjugated antibodies. In 1 hour cells were PBS washed and ELISA was developed by using TMB peroxidase substrate solution (Pierce, Rockford, IL). After 30-min incubation, plates were read at $450 \mathrm{~nm}$ on a plate reader.

LDH assay. Cells were grown in 96-well plates with concentration of $1 \times 10^{4}$ cells per well. N10 glioma cells were treated with either mock, IgG or IL13-MDs for $1 \mathrm{hr}$ followed by exposure to magnetic fields of $0 \sim 60 \mathrm{~Hz}(90 \mathrm{Oe}$ ) for $10 \mathrm{~min}$. After magnetic field application, $50 \mu \mathrm{l}$ of LDH substrate solution was added to each well with following incubation for 30 min at room temperature. Next, $50 \mu \mathrm{l}$ of stop solution was added to each well in the plate and read on a micro-ELISA reader (Biotec 800, ISC Bioexpress, USA) at a wavelength of 490 $\mathrm{nm}$. The percentage of cytotoxicity was calculated with control wells containing cell culture medium without any treatment. $\mathrm{LDH}$ leakage $(\%)=\left[\mathrm{A}_{\text {test }}\right] /\left[\mathrm{A}_{\text {control }}\right] \times 100$, where $\left[\mathrm{A}_{\text {test }}\right]$ is the absorbance of the test sample and [ $\left.\mathrm{A}_{\text {control }}\right]$ is the absorbance of the untreated control sample. TUNEL assay. $2 \times 10^{4}$ N10 glioma cells were seeded and grown on polylysine-coated coverslips. Then, N10 glioma cells were incubated with either mAb or negative control IgG1-modified MDs for $1 \mathrm{hr}$ followed by exposure to magnetic fields of $0 \sim 130 \mathrm{Oe}(20 \mathrm{~Hz})$ for $10 \mathrm{~min}$. In control experiment $20 \mu \mathrm{M}$ caspase Inhibitor Z-VAD-FMK was added. The cells were then washed with serum-free DMEM media and fixed with $1 \%$ Formalin-PBS solution (Boston Bioproduct, Boston, USA). After washing 3 times with $500 \mu \mathrm{ml}$ PBS, treated N10 cells on the coverslips were processed by a TUNEL assay kit according to the vendor recommendations. Briefly, the slides after treatment were fixed and then labeled by incubation with the reaction mixture containing TdT and digoxigenin-conjugated dUTP for $1 \mathrm{hr}$ at $37^{\circ} \mathrm{C}$. Labeled DNA was visualized with peroxidase-conjugated anti-digoxigenin antibody with $3,3^{\prime}$-diaminobenzidene as the chromagen.

\section{Conclusion \& outlook}

The magnetic properties, fabrication, and biofunctionalized of the lithographically defined microdisks with spin vortex state were described. It was shown that targeted magnetomechanical cancer cell destruction can be achieved using properly biofunctionalized microdisks subjected to an unprecedentedly slow magnetic field. In these experiments an external energy power supplied to the cell cultures was $\sim 10^{5}$ times smaller than the typical hyperthermia use of magnetic nanoparticles (Hergt, 2006; Liu, 2007; Goya, 2008). The weak fields mean that the method has great potential for a low cost, large working volume and minimally invasive therapies. Besides the field frequencies and amplitude, the most striking difference is that in our approach allows the energy is delivered directly to the cell, e.g. potentially without damaging the surrounding normal cells or tissues. Furthermore, if was found that the microdisk-induced mechanical stimulus results into intracellular signal 
transduction and amplification, causing initiation of programmed cell death. Future efforts could focussed on (1) testing different cancer cell line, including human cell line, (2) use the microdisk in in vivo models by combining low-frequency (magnetomechanical destruction) and high frequency (thermal ablation) fields, and (3) exploring scalability of this approach down to $\sim 100 \mathrm{~nm}$ dimentions.

\section{Acknowledgements}

We thank our collaborators Drs. S. D. Bader, R. Divan, D.-H. Kim, J. Pearson, T. Rajh, V. G. Yefremenko from Argonne, Drs. V. Bindokas, M. S. Lesniak and I. V. Ulasov from the University of Chicago for continued involvement and interest to this project. Work at Argonne and its Center for Nanoscale Materials and Electron Microscopy Center is supported by the US Department of Energy Office of Science, Basic Energy Sciences, under contract No DE-AC02-06CH11357.

\section{References}

Ade, H. \& Stoll, H. Near-edge X-ray absorption fine-structure microscopy of organic and magnetic materials. Nature Materials vol. 8, pp. 281-290 (2009).

Boehning, D., Patterson, R. L., Sedaghat, L., Glebova, N. O., Kurosaki, T. \& Snyder S. H. Cytochrome c binds to inositol $(1,4,5)$ trisphosphate receptors, amplifying calciumdependent apoptosis. Nature Cell Biology vol. 5, pp. 1051 - 1061 (2003).

Clapham D. E., Calcium signaling. Cell 80, 259-268 (1995).

Chung, S.-H.; McMichael, R.; Pierce, D \& J. Unguris, Phase diagram of magnetic nanodisks measured by scanning electron microscopy with polarization analysis. Physical Review B, vol. 81, 024410 (2010).

Cowburn, R. P., Koltsov, D. K., Adeyeye, A. O., and Welland, M. E. Single-Domain Circular Nanomagnets, Phys. Rev. Lett. Vol. 83, pp. 1042-1045 (1999).

Da, K., Shiyama, K., Naka, R., Hiyama, A. \& Anishi, T. GFAP-positive human glioma cell lines: no. 10, no.11. Human Cell vol. 3, pp. 251-256 (1990).

Debinski, W., Gibo, D., Hulet, S., Connor, J. \& Gillespie, G. Receptor for interleukin 13 is a marker and therapeutic target for human high-grade gliomas. Cancer Res. Vol.5, pp. 985-990 (1999).

Demokritov, S.; Hillebrands B. \& Slavin, N. Brillouin light scattering studies of confined spin waves: linear and non-linear confiment. Physics Reports, vol. 348 pp. 441-489 (2001).

Dobson, J. Remote control of cellular behaviour with magnetic nanoparticles. Nature Nanotechnology 3, 139-148 (2008).

Donahue, M. \& Porter, D. Object Oriented Micromagnetic Framework (OOMMF) User's Guide, Version 1.0, Interagency Report NIST IR 6376, National Institute of Standards \& Technology, Gaithersburg, MD, 1999.

Duchen. M. R. Mitochondria and calcium: from cell signalling to cell death. eburnal of Physiology 529, 57-68 (2000).

Ferrari, M. Cancer nanotechnology: opportunities and challenges. Nature Reviews Cancer 5, 161-171 (2005).

Fu, A. ; Hu, W. ; Xu, L. ; Wilson, R. ; Yu, H., Osterfeld, S.; Gambhir, S. \& Wang, S. Proteinfunctionalized synthetic antiferromagnetic nanoparticles for biomolecule detection and magnetic manipulation, Angew. Chem. Int. Ed., vol. 48, pp. 1620. (2009). 
Gao, J.; Gu, H. \& XU, B. Multifunctional magnetic nanoparticles: design, synthesis, and biomedical applications. Accounts of chemical research, Vol. 42, No. 8, pp. 1097-1107, 2009.

Goya, G. F., Grazu, V. \& Ibarra, M. R. Magnetic nanoparticles for cancer therapy. Current Nanoscience 4, 1-16 (2008).

Guharay, F. \& Sachs, F. J. Stretch-activated single ion channel currents in tissue-cultured embryonic chick skeletal muscle. Physiol. (London) 352, 685-701 (1984).

Gupta, A. K. \& Gupta, M. Synthesis and surface engineering of iron oxide nanoparticles for biomedical applications. Biomaterials 26, 3995-4021 (2005).

Guslienko, K.; Novosad, V.; Otani, Y.; Shima, H. \& and Fukamichi, K. Magnetization reversal due to vortex nucleation, displacement, and annihilation in submicron ferromagnetic dot arrays. Phys. Rev. B vol. 65, p. 024414 (2001).

Hergt, R.; Dutz, S.; Muller, R. \& Zeisberger, M. Magnetic particle hyperthermia: nanoparticle magnetism and materials development for cancer therapy. I Phys. Condens. Matter. Vol. 18, pp. 2919 -2923 (2006).

Hu, W.; Wilson, R.; Koh, A.; Fu, A.; Faranesh; A. Z.; Earhart, C.; Osterfeld, S.; Han, S.-J.; Xu, L.; Guccione, S.; Sinclair, R.; \& Wang, S. High-Moment Antiferromagnetic Nanoparticles with Tunable Magnetic Properties. Advanced Materials, vol., pp. 14791483 (2008).

Kasibhatla S, \& Tseng, B. Why Target Apoptosis in Cancer Treatment? Molecular Cancer Theropy, vol. 2, pp. 573-580 (2003).

Kawakami, K., Kawakami, M., Snoy, P. J., Husain, S. R. \& Puri, R. In vivo overexpression of IL-13 receptor $\alpha 2$ chain inhibits tumorigenicity of human breast and pancreatic tumors in immunodeficient mice. el Exp. Med. 194, 1743-1754 (2001).

Kim, D.-H.; Rozhkova, E.; Ulasov, I.; Bader, S.; Rajh, T., Lesniak, M. \& Novosad, V. Biofunctionalized magnetic-vortex microdiscs for targeted cancer-cell destruction. Nature Materials, vol.9, pp. 165 - 171 (2010).

Krishnan, K. Biomedical nanomagnetics: a spin through possibilities in imaging, diagnostics, and therapy. IEEE Transactions in Magnetics, vol. 46, No. 7, pp. 2523- 2558, (2010).

Liu, X.; Novosad, V.; Rozhkova, E.; Chen, H.; Yeferemenko, V.; Pearson, J.; Torno, M.; Bader, S. \& Rosengart, A. Surface Functionalized Biocompatible Magnetic Nanospheres for Cancer Hyperthermia, IEEE Transactions on Magnetics, vol. 43, issue 6, pp. 2462-2464 (2007).

Mair, L. ; Ford, K. ; Alam, M.; Kole, R.; Fisher, \&M. Superfine, R. Size-uniform 200 nm particles: Fabrication and Application to Magnetofection. I Biomed Nanotechnol. Vol. 5, pp. 182-191, (2009).

Mannix, R. J., Kumar, S., Cassiola, F., Montoya-Zavala, M., Feinstein, E., Prentiss, M. \& Ingber D. E., Nanomagnetic actuation of receptor-mediated signal transduction. Nature Nanotechnology vol. 3, pp. 36-40 (2007).

Martinac, B. Mechanosensitive ion channels: molecules of mechanotransduction. I Cell Science 117, 2449-2460 (2004).

Mattson, M. P. \& Chan, S. L. Calcium orchestrates apoptosis. Nature Cell Biology 5, 1041 1043 (2003).

Mpoke, S. S. \& Wolfe, J. Differential Staining of Apoptotic Nuclei in Living Cells: Application to macronuclear elimination in tetrahymena. I Histochem. Cytochem. Vol. 45, 675-684 (1997). 
Muller, D., Helenius J., Alsteens D, \& Dufrêne Y. Force probing surfaces of living cells to molecular resolution. Nature Chem. Biology, vol. 5, 383-391 (2009).

Nel, A.; Mädler, L.; Valegol, D.; Xia. T.; Hoek, E.; Somasundaran, P.; Klaessig, F.; Castranova, V. \& Thompson, M. Understanding biophysicochemical interactions at the nano-bio interface. Nature Materials, vol. 8, pp. 2442-15 (2009).

Novosad, V.; Guslienko, K.; Shima, H.; Otani, Y.; Kim, S.; Fukamichi,K.; Kikuchi N.; Kitakami, O. \& Shimada, Y. Effect of interdot magnetostatic interaction on magnetization reversal in circular dot arrays. Phys. Rev. B, vol. 65, 060402 (2002).

Pankhurst, Q.; Thanh, N.; Jones, S. \& Dobson, J. Progress in applications of magnetic nanoparticles in biomedicine. I Phys. D: Appl. Phys. Vol. 42, 224001 (2009).

Rozhkova, E.; Ulasov, I.; Lai, B.; Dimitrijevic, N.; Lesniak, M. \& Rajh, T. A High-Performance Nanobio Photocatalyst for Targeted Brain Cancer Therapy. NanoLetters 9, 3337 3342 (2009).

Scholz,W.; Guslienko, K.; Novosad, V.; Suess,D.; Schrefl, T.; Chantrell, R. \& Fidler, J. Transition from single-domain to vortex state in soft magnetic cylindrical nanodots. burnal of Magnetism and Magnetic Materials. Vol. 266, No. 1-2, pp. 155-163, (2003).

Shinjo, T.; Okuno, T.; Hassdorf, R.; Shigeto, K. \& Ono, T. Magnetic vortex core observation in circular dots of permalloy. Science 289, 930-933 (2000).

Sen, S., Subramanian, S. \& Discher, D. E. Indentation and adhesive probing of a cell membrane with AFM: theoretical model and experiments, Biophysical eburnal, 89, 3203-3213 (2005).

T. Vaughan, J. Weaver, Energetic constraints on the creation of cell membrane pores by magnetic particles. Biophysical eburnal, vol. 71, pp. 616-622 (1996).

Wachowiak, A.; Wiebe, J.; Bode, M.; Pietzsch, O.; Morgenstern, M. \& Wiesendanger, R. Direct observation of internal spin structure of magnetic vortex cores. Science 298, 577-580 (2002).

Wang, S, \& Li, G. Advances in giant magnetoresistance biosensors with magnetic manoparticle tags: review and outlook. IEEE Trans. Mag. Vol. 44, 1687 (2008).

Yakovlev, A.G., Wang, G., Stoica, B. A., Simbulan-Rosenthal, C. M., Yoshihara, K. \& Smulson, M. E. Role of DNAS1L3 in Ca2+- and Mg2+-dependent cleavage of DNA into oligonucleosomal and high molecular mass fragments. Nucleic Acids Research 27, 1999-2005 (1999).

Zabow, G. ; Dodd, S. ; Moreland, J. \& Koretsky, A. Micro-engineered local field control for high-sensitivity multispectral MRI. Nature, vol. 453, 1058 (2008).

Zabow, G. ; Dodd, S. ; Moreland, J. \& Koretsky, A. The fabrication of uniform cylindrical nanoshells and their use as spectrally tunable MRI contrast agents. Nanotechnology, vol. 20, 385301 (2009). 


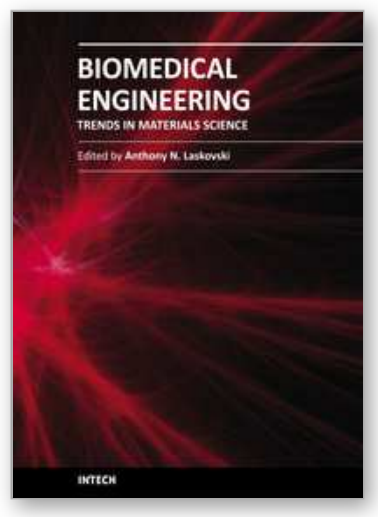

\author{
Biomedical Engineering, Trends in Materials Science \\ Edited by Mr Anthony Laskovski
}

ISBN 978-953-307-513-6

Hard cover, 564 pages

Publisher InTech

Published online 08, January, 2011

Published in print edition January, 2011

Rapid technological developments in the last century have brought the field of biomedical engineering into a totally new realm. Breakthroughs in materials science, imaging, electronics and, more recently, the information age have improved our understanding of the human body. As a result, the field of biomedical engineering is thriving, with innovations that aim to improve the quality and reduce the cost of medical care. This book is the second in a series of three that will present recent trends in biomedical engineering, with a particular focus on materials science in biomedical engineering, including developments in alloys, nanomaterials and polymer technologies.

\title{
How to reference
}

In order to correctly reference this scholarly work, feel free to copy and paste the following:

Valentyn Novosad and Elena A. Rozhkova (2011). Ferromagnets-Based Multifunctional Nanoplatform for Targeted Cancer Therapy, Biomedical Engineering, Trends in Materials Science, Mr Anthony Laskovski (Ed.), ISBN: 978-953-307-513-6, InTech, Available from: http://www.intechopen.com/books/biomedical-engineeringtrends-in-materials-science/ferromagnets-based-multifunctional-nanoplatform-for-targeted-cancer-therapy

\section{INTECH}

open science | open minds

\section{InTech Europe}

University Campus STeP Ri

Slavka Krautzeka 83/A

51000 Rijeka, Croatia

Phone: +385 (51) 770447

Fax: +385 (51) 686166

www.intechopen.com

\section{InTech China}

Unit 405, Office Block, Hotel Equatorial Shanghai

No.65, Yan An Road (West), Shanghai, 200040, China

中国上海市延安西路65号上海国际贵都大饭店办公楼405单元

Phone: +86-21-62489820

Fax: +86-21-62489821 
(C) 2011 The Author(s). Licensee IntechOpen. This chapter is distributed under the terms of the Creative Commons Attribution-NonCommercialShareAlike-3.0 License, which permits use, distribution and reproduction for non-commercial purposes, provided the original is properly cited and derivative works building on this content are distributed under the same license. 